\title{
Efficient Multi-party Computation over Rings
}

\author{
Ronald Cramer ${ }^{1}$, Serge Fehr ${ }^{1}$, Yuval Ishai ${ }^{2}$, and Eyal Kushilevitz ${ }^{2}$ \\ 1 BRICS $^{\star \star \star}$, Department of Computer Science, Århus University, Denmark \\ $\{$ cramer, fehr\}@brics.dk \\ 2 Computer Science Department, Technion, Israel \\ \{yuvali, eyalk\}@cs.technion.ac.il
}

\begin{abstract}
Secure multi-party computation (MPC) is an active research area, and a wide range of literature can be found nowadays suggesting improvements and generalizations of existing protocols in various directions. However, all current techniques for secure MPC apply to functions that are represented by (boolean or arithmetic) circuits over finite fields. We are motivated by two limitations of these techniques:

- Generality. Existing protocols do not apply to computation over more general algebraic structures (except via a brute-force simulation of computation in these structures).

- EFficiency. The best known constant-round protocols do not efficiently scale even to the case of large finite fields.

Our contribution goes in these two directions. First, we propose a basis for unconditionally secure MPC over an arbitrary finite ring, an algebraic object with a much less nice structure than a field, and obtain efficient MPC protocols requiring only a black-box access to the ring operations and to random ring elements. Second, we extend these results to the constant-round setting, and suggest efficiency improvements that are relevant also for the important special case of fields. We demonstrate the usefulness of the above results by presenting a novel application of MPC over (non-field) rings to the round-efficient secure computation of the maximum function.
\end{abstract}

\section{Introduction}

BACKGROUND. The goal of secure multi-party computation (MPC), as introduced by Yao [37, is to enable a set of players to compute an arbitrary function $f$ of their private inputs. The computation must guarantee the correctness of the result while preserving the privacy of the players' inputs, even if some of the players are corrupted by an adversary and misbehave in an arbitrary malicious way. Since the initial plausibility results in this area 3824610 , much effort has been put into enhancing these results, and nowadays there is a wide range of literature treating issues like improving the communication complexity (e.g., [22, 23[26]) or the round complexity (e.g., [15/28] ), and coping with more powerful (e.g., 3498) or more general (e.g., 25 19,13]) adversaries.

$\star \star \star$ Basic Research in Computer Science (www.brics.dk), funded by the Danish National Research Foundation. 
A common restriction on all these results is that the function $f$ is always assumed to be represented by an arithmetic circuit over a finite field, and hence all computations "take place" in this field. Thus, it is natural to ask whether MPC can also be efficiently implemented over a richer class of structures, such as arbitrary finite rings. This question makes sense from a theoretical point of view, where it may be viewed as a quest for minimizing the axioms on which efficient secure MPC can be based, but also from a practical point of view, since a positive answer would allow greater freedom in the representation of $f$, which in turn can lead to efficiency improvements. Unfortunately, general rings do not enjoy some of the useful properties of fields on which standard MPC protocols rely: non-zero ring elements may not have inverses (in fact, a ring may even not contain 1 , in which case no element is invertible), there might exist zero-divisors, and the multiplication may not be commutative. Indeed, already over a relatively "harmless" ring like $\mathbb{Z}_{m}$, Shamir's secret sharing scheme [36], which serves as the standard building block for MPC, is not secure a-priori. For instance, if $m$ is even and if $p(X)$ is a polynomial over $\mathbb{Z}_{m}$ hiding a secret $s$ as its free coefficient, then the share $s_{2}=p(2)$ is odd if and only if the secret $s$ is odd. Thus, even the most basic tools for secure MPC have to be modified before applying them to the case of rings. A step in this direction was taken in 1815, where additively homomorphic secret sharing schemes for arbitrary Abelian groups have been proposed. However, this step falls short of providing a complete solution to our problem (which in particular requires both addition and multiplication of shared secrets), and so the question of MPC over rings remains unanswered.

An additional limitation of current MPC techniques which motivates the current work is related to the efficiency of constant-round protocols. Without any restriction on the number of rounds, most protocols from the literature generalize smoothly to allow arithmetic computation over arbitrary finite fields. This is particularly useful for the case of "numerical" computations, involving integers or (finite-precision) reals; indeed, such computations can be naturally embedded into fields of a sufficiently large characteristic. However, in the constant-round setting the state of affairs is quite different. All known protocols for efficiently evaluating a circuit in a constant number of rounds [38|5 11/32] are based on Yao's garbled circuit construction, which does not efficiently scale to arithmetic circuits over large fields 1 The only constant-round protocols in the literature which do efficiently scale to arithmetic computation over large fields apply to the weaker computational models of formulas [1] or branching programs [29], and even for these models their complexity is (at least) quadratic in the representation size. Hence, there are no truly satisfactory solutions to the problem of constant-round MPC over general fields, let alone general rings.

\footnotetext{
${ }^{1}$ It is obviously possible to apply the brute-force approach of simulating each field operation by a boolean circuit computing it. However, this approach is unsatisfactory both from a theoretical point of view (as its complexity grows super-linearly in the length of a field element) and from a practical point of view. The same objection applies to the implementation of ring operations using field or boolean operations.
} 
OUR RESULTS. In this paper, we propose a basis for obtaining unconditionally secure MPC over arbitrary finite rings. In particular, we present an efficient MPC protocol that requires only black-box access to the ring operations (addition, subtraction, and multiplication) and the ability to sample random ring elements. It is perfectly secure with respect to an active adversary corrupting up to $t<n / 3$ of the $n$ players, and its complexity is comparable to the corresponding fieldbased solutions. This is a two-fold improvement over the classical field-based $\mathrm{MPC}$ results. It shows that MPC can be efficiently implemented over a much richer class of structures, namely arbitrary finite rings, and it shows that there exists in fact one "universal" protocol that works for any finite ring (and field). Finally, the tools we provide can be combined with other work on MPC, and hence expand a great body of work on MPC to rings.

On the constant-round front, we make two distinct contributions. First, we show that the feasibility of MPC over black-box rings carries over to the constantround setting 2 To this end, we formulate and utilize a garbled branching program construction, based on a recent randomization technique from [29]; however, as the algebraic machinery which was originally used in its analysis does not apply to general rings, we provide a combinatorially-oriented presentation and analysis which may be of independent interest. As a second contribution, we suggest better ways for evaluating natural classes of arithmetic formulas and branching programs in a constant number of rounds. In particular, we obtain protocols for small-width branching programs and balanced formulas in which the communication complexity is nearly linear in their size. The former protocols are based on the garbled branching program construction, and the latter on a combination of a complexity result from 12 with a variant of randomization technique from [3]. While the main question in this context (namely, that of obtaining efficient constant-round protocols for arithmetic circuits) remains open, our techniques may still provide the best available tools for efficiently realizing "numerical" MPC tasks that arise in specific applications. Furthermore, these techniques may also be beneficial in the two-party setting of 35 (via the use of a suitable homomorphic encryption scheme) and in conjunction with computationally-secure MPC (using, e.g., [14]).

We conclude with an example for the potential usefulness of secure MPC over non-field rings. Specifically, we show how to efficiently compute the maximum of $n$ integers with better round complexity than using alternative approaches.

Organization. Section 2 deals with the model. The main body of the paper has two parts corresponding to our two main contributions: the first deals with general MPC over rings (Section [3) and the other concentrates on constantround protocols (Section 4). Finally, in Section 5 we describe an application of MPC over non-field rings. A longer version of this paper, which in particular contains some proofs that were omitted from this version, can be found in [16].

\footnotetext{
${ }^{2}$ This is not clear a-priori, and in fact most randomization techniques used in the context of constant-round MPC (e.g., $[1,20|3| 28]$ ) clearly do not apply to this more general setting.
} 


\section{Model}

We consider the secure-channels model, as introduced in [6]10], where a set $\mathcal{P}=\left\{P_{1}, \ldots, P_{n}\right\}$ of $n$ players is connected by bilateral, synchronous, reliable secure channels. For the case of constant-round secure computation, a broadcast channel is also assumed to be available, while it has to be implemented otherwise. Our goal is to obtain a protocol for securely computing a function given by an arithmetic circuit over an arbitrary ring $R$. (In Section 4 we will also be interested in functions represented by formulas and branching programs over a ring $R$.) By default, we consider unconditional or perfect security against an adaptive, active adversary. The reader is referred to, e.g., 7] for a definition of secure protocols in this setting. Such a protocol is black-box if: (1) its description is independent of $R$ and it only makes black-box calls to the ring operations (addition, subtraction and multiplication) and to random ring elements; and (2) its security holds regardless of the underlying ring $R$, in the sense that each adversary attacking the protocol admits a simulator having only a black-box access to $R$.

\section{Multi-party Computation over Rings}

\subsection{Mathematical Preliminaries}

We assume the reader to be familiar with basic concepts of group and ring theory. However, we also make use of the notions of a module and of an algebra, which we briefly introduce here. Let $\Lambda$ be a commutative ring with 1 . An (additive) Abelian group $G$ is called a $\Lambda$-module if a number multiplication $\Lambda \times G \rightarrow G,(\lambda, a) \mapsto \lambda \cdot a$ is given such that $1 \cdot a=a, \lambda \cdot(a+b)=(\lambda \cdot a)+(\lambda \cdot b),(\lambda+\mu) \cdot a=(\lambda \cdot a)+(\mu \cdot a)$ and $(\lambda \cdot \mu) \cdot a=\lambda \cdot(\mu \cdot a)$ for all $\lambda, \mu \in \Lambda$ and $a, b \in G$. Hence, loosely speaking, a module is a vector space over a ring (instead of over a field). An arbitrary ring $R$ is called a $\Lambda$-algebra if (the additive group of) $R$ is a $\Lambda$-module and $(\lambda \cdot a) \cdot b=\lambda \cdot(a \cdot b)=a \cdot(\lambda \cdot b)$ holds for all $\lambda \in \Lambda$ and $a, b \in R$. For example, every Abelian group $G$ is a $\mathbb{Z}$-module and every ring $R$ is a $\mathbb{Z}$-algebra; the number multiplication is given by $0 \cdot a=0, \lambda \cdot a=a+\cdots+a$ ( $\lambda$ times $)$ if $\lambda>0$, and $\lambda \cdot a=-((-\lambda) \cdot a)$ if $\lambda<0$. We also write $\lambda a$ or $a \lambda$ instead of $\lambda \cdot a$.

\subsection{Span Programs over Rings and Linear Secret Sharing}

Monotone span programs over (finite) fields were introduced in [30] and turned out to be in a one-to-one correspondence to linear secret sharing schemes (over finite fields). This notion was extended in [15] to monotone span programs over (possibly infinite) rings, and it was shown that integer span programs, i.e. span programs over $\mathbb{Z}$, have a similar correspondence to black-box secret sharing (over arbitrary Abelian groups). We briefly recall some definitions and observations.

Definition 1. A subset $\Gamma$ of the power set $2^{\mathcal{P}}$ of $\mathcal{P}$ is called an access structure on $\mathcal{P}$ if $\emptyset \notin \Gamma$ and if $\Gamma$ is closed under taking supersets: $A \in \Gamma$ and $A^{\prime} \supseteq A$ implies that $A^{\prime} \in \Gamma$.A subset $\mathcal{A}$ of $2^{\mathcal{P}}$ is called an adversary structure on $\mathcal{P}$ if its complement $\mathcal{A}^{c}=2^{\mathcal{P}} \backslash \mathcal{A}$ is an access structure. 
Let $\Lambda$ be an arbitrary (not necessarily finite) commutative ring with 1 . Consider a matrix $M$ over $\Lambda$ with, say, $d$ rows and $e$ columns (this will be denoted as $\left.M \in \Lambda^{d \times e}\right)$, a labeling function $\psi:\{1, \ldots, d\} \rightarrow \mathcal{P}$ and the target vector $\varepsilon=(1,0, \ldots, 0)^{T} \in \Lambda^{e}$. The function $\psi$ labels each row of $M$ with a number corresponding to one of the players. If $A \subseteq \mathcal{P}$ then $M_{A}$ denotes the restriction of $M$ to those rows $i$ with $\psi(i) \in A$, and, similarly, if $\mathbf{x}$ denotes an arbitrary $d$-vector then $\mathbf{x}_{A}$ denotes the restriction to those coordinates $i$ with $\psi(i) \in A$. In case $A=\left\{P_{i}\right\}$, we write $M_{i}$ and $\mathbf{x}_{i}$ instead of $M_{A}$ and $\mathbf{x}_{A}$. Finally, im $(\cdot)$ denotes the image and $\operatorname{ker}(\cdot)$ the kernel (or null-space) of a matrix.

Definition 2. Let $\mathcal{M}=(\Lambda, M, \psi, \varepsilon)$ be a quadruple as above, and let $\Gamma$ be an access structure on $\mathcal{P}$. Then, $\mathcal{M}$ is called a (monotone) ${ }^{3}$ span program (over $\Lambda$ ) for the access structure $\Gamma$, or, alternatively, for the adversary structure $\mathcal{A}=\Gamma^{c}$, if for all $A \subseteq \mathcal{P}$ the following holds.

- If $A \in \Gamma$, then $\varepsilon \in \operatorname{im}\left(M_{A}^{T}\right)$, and

- if $A \notin \Gamma$, then there exists $\kappa=\left(\kappa_{1}, \ldots, \kappa_{e}\right)^{T} \in \operatorname{ker}\left(M_{A}\right)$ with $\kappa_{1}=1$.

If $\Lambda=\mathbb{Z}$ then $\mathcal{M}$ is called an integer span program, ISP for short. Finally, $\operatorname{size}(\mathcal{M})$ is defined as $d$, the number of rows of $M$.

By basic linear algebra, the existence of $\kappa \in \operatorname{ker}\left(M_{A}\right)$ with $\kappa_{1}=1$ implies that $\varepsilon \notin \operatorname{im}\left(M_{A}^{T}\right)$, however the other direction generally only holds if $\Lambda$ is a field.

Let $G$ be an arbitrary finite Abelian group that can be seen as a $\Lambda$-module. As a consequence, it is well defined how a matrix over $\Lambda$ acts on a vector with entries in $G$. Then, a span program $\mathcal{M}=(\Lambda, M, \psi, \varepsilon)$ for an access structure $\Gamma$ gives rise to a secret sharing scheme for secrets in $G$ :

To share $s \in G$, the dealer chooses a random vector $\mathbf{b}=\left(b_{1}, \ldots, b_{e}\right)^{T} \in G^{e}$ of group elements with $b_{1}=s$, computes $\mathbf{s}=M \mathbf{b}$ and, for every player $P_{i} \in \mathcal{P}$, hands $\mathbf{s}_{i}$ (privately) to $P_{i}$. This is a secure sharing of $s$, with respect to the access structure $\Gamma$. Namely, if $A \in \Gamma$ then there exists an ( $A$-dependent) vector $\boldsymbol{\lambda}$, with entries in $\Lambda$, such that $M_{A}^{T} \boldsymbol{\lambda}=\boldsymbol{\varepsilon}$. It follows that $s$ can be reconstructed from $\mathbf{s}_{A}$ by $\mathbf{s}_{A}^{T} \boldsymbol{\lambda}=\left(M_{A} \mathbf{b}\right)^{T} \boldsymbol{\lambda}=\mathbf{b}^{T} M_{A}^{T} \boldsymbol{\lambda}=\mathbf{b}^{T} \boldsymbol{\varepsilon}=s$. On the other hand, if $A \notin \Gamma$ then there exists an $\left(A\right.$-dependent) vector $\boldsymbol{\kappa} \in \Lambda^{e}$ with $M_{A} \boldsymbol{\kappa}=\mathbf{0}$ and $\kappa_{1}=1$. For arbitrary $s^{\prime} \in G$ define $\mathbf{s}^{\prime}=M\left(\mathbf{b}+\boldsymbol{\kappa}\left(s^{\prime}-s\right)\right)$. The secret defined by $\mathbf{s}^{\prime}$ equals $s^{\prime}$, while on the other hand $\mathbf{s}_{A}^{\prime}=\mathbf{s}_{A}$. Hence, the assignment $\mathbf{b}^{\prime}=\mathbf{b}+\boldsymbol{\kappa}\left(s^{\prime}-s\right)$ provides a bijection between the random coins (group elements) consistent with $\mathbf{s}_{A}$ and $s$ and those consistent with $\mathbf{s}_{A}$ and $s^{\prime}$. This implies (perfect) privacy.

Note that since every Abelian group $G$ is a $\mathbb{Z}$-module, an $I S P$ gives rise to a black-box secret sharing scheme 15]. Furthermore, the above applies in particular to (the additive group of) a ring $R$ which can be seen as a $\Lambda$-algebra.

\subsection{Multiplicative Span Programs and Secure MPC}

The multiplication property for a span program over a field has been introduced in 13 . It essentially requires that the product of two shared secrets can be written as a linear combination of locally computable products of shares. However,

\footnotetext{
${ }^{3}$ Since we consider only monotone span programs, we omit the word "monotone".
} 
in our setting (where, given the span program, it is not clear from what ring $R$ the secret and the shares will be sampled), we define the multiplication property as a sole property of the span program.

Let $\Lambda$ be a commutative ring with 1 , and let $\mathcal{M}=(\Lambda, M, \psi, \varepsilon)$ be a span program over $\Lambda$ for an adversary structure $\mathcal{A}$.

Definition 3. The span program $\mathcal{M}$ is called multiplicative if there exists a block-diagonal matrix $D \in \Lambda^{d \times d}$ such that $M^{T} D M=\varepsilon \varepsilon^{T}$, where block-diagonal is to be understood as follows. Let the rows and columns of $D$ be labeled by $\psi$, then the non-zero entries of $D$ are collected in blocks $D_{1}, \ldots, D_{n}$ such that for every $P_{i} \in \mathcal{P}$ the rows and columns in $D_{i}$ are labeled by $P_{i}$.

$\mathcal{M}$ is called strongly multiplicative if, for every player set $A \in \mathcal{A}, \mathcal{M}$ restricted to the complement $A^{c}$ of $A$ is multiplicative.

As in the case of span programs over fields (see [13]), for every adversary structure $\mathcal{A}$ there exists a (strongly) multiplicative span program $\mathcal{M}$ over $\Lambda$ for $\mathcal{A}$ if and only if $\mathcal{A}$ is $Q^{2}\left(Q^{3}\right)$, meaning that no two (three) sets of $\mathcal{A}$ cover the whole player set $\mathcal{P}[25]$. Furthermore, there exists an efficient procedure to transform any span program $\mathcal{M}$ over $\Lambda$ for a $Q^{2}$ adversary structure $\mathcal{A}$ into a multiplicative span program $\mathcal{M}^{\prime}$ (over $\Lambda$ ) for the same adversary structure $\mathcal{A}$, such that the size of $\mathcal{M}^{\prime}$ is at most twice the size of $\mathcal{M}$. 4

Similarly to the field case, the multiplication property allows to securely compute a sharing of the product of two shared secrets. Indeed, let $R$ be a finite ring which can be seen as a $\Lambda$-algebra, and let $\mathbf{s}=M \mathbf{b}$ and $\mathbf{s}^{\prime}=M \mathbf{b}^{\prime}$ be sharings of two secrets $s, s^{\prime} \in R$. Then, the product $s s^{\prime}$ can be written as $s s^{\prime}=\mathbf{b}^{T} \varepsilon \varepsilon^{T} \mathbf{b}^{\prime}=\mathbf{b}^{T} M^{T} D M \mathbf{b}^{\prime}=(M \mathbf{b})^{T} D M \mathbf{b}^{\prime}=\mathbf{s}^{T} D \mathbf{s}^{\prime}=\sum_{i} \mathbf{s}_{i}^{T} D_{i} \mathbf{s}_{i}^{\prime}$, i.e., by the special form of $D$, as the sum of locally computable values. Hence the multiplication protocol from [23] can be applied: To compute securely a sharing $\mathbf{s}^{\prime \prime}=M \mathbf{b}^{\prime \prime}$ of the product $s s^{\prime}$, every player $P_{i}$ shares $p_{i}=\mathbf{s}_{i}^{T} D_{i} \mathbf{s}_{i}^{\prime}$, and then every player $P_{i}$ adds up its shares of $p_{1}, \ldots, p_{n}$, resulting in $P_{i}^{\prime}$ 's share $\mathbf{s}_{i}^{\prime \prime}$ of $s s^{\prime}$.

Given a multiplicative span program over $\Lambda$ for a $Q^{2}$ adversary structure $\mathcal{A}$ (where the multiplication property can always be achieved according to a remark above), it follows that if $R$ is a $\Lambda$-algebra, then any circuit over $R$ can be computed securely with respect to a passive adversary that can (only) eavesdrop the players of an arbitrary set $A \in \mathcal{A}$. Namely, every player shares its private input(s) using the secret sharing scheme described in Section 3.2 , and then the circuit is securely evaluated gate by gate, the addition gates non-interactively based on the homomorphic property of the secret sharing scheme, and the multiplication gates using the above mentioned multiplication protocol. Finally, the (shared) result of the computation is reconstructed. We sketch in Section 3.4 how to achieve security against an active $Q^{3}$ adversary. Note that a broadcast channel can be securely implemented using, e.g., [21]. All in all, this proves

\footnotetext{
${ }^{4}$ A similar result concerning the strong multiplication property is not known to exist, not even in the field case.
} 
Theorem 1. Let $\Lambda$ be a commutative ring with 1 , and let $\mathcal{M}$ be a (strongly 5 multiplicative span program over $\Lambda$ for a $Q^{3}$ adversary structure $\mathcal{A}$. Then there exists an $\mathcal{A}$-secure $M P C$ protocol to evaluate any arithmetic circuit $C$ over an arbitrary finite ring $R$ which can be seen as a $\Lambda$-algebra.

Concerning efficiency, the communication complexity of the MPC protocol (in terms of the number of ring elements to be communicated) is polynomial in $n$, in the size of $\mathcal{M}$, and in the number of multiplication gates in $C$.

Corollary 1. Let $\mathcal{M}$ be a (strongly) multiplicative ISP for a $Q^{3}$ adversary structure $\mathcal{A}$. Then there exists an $\mathcal{A}$-secure black-box $M P C$ protocol to evaluate any arithmetic circuit $C$ over an arbitrary finite ring $R$.

The black-box MPC result from Corollary 1 exploits the fact that every ring $R$ is a $\mathbb{Z}$-algebra 6 If, however, additional information about $R$ is given, it might be possible to view $R$ as an algebra over another commutative ring $\Lambda$ with 1 . For example, if the exponent $\ell$ of (the additive group of) $R$ is given, then we can exploit the fact that $R$ is an algebra over $\Lambda=\mathbb{Z}_{\ell}$. In many cases, this leads to smaller span programs and thus to more efficient MPC protocols than in the black-box case. For instance, if the exponent of $R$ is a prime $p$ then $R$ is an algebra over the field $\mathbb{F}_{p}$, and we can apply standard techniques to derive span programs over $\mathbb{F}_{p}$ (or an extension field). If the exponent $\ell$ is not prime but, say, square-free, we can use Chinese Remainder Theorem to construct suitable span programs. See also Proposition $\square$ for the case of a threshold adversary structure.

\subsection{Adversary Achieving Security against an Active Adversary}

Following the paradigm of [13], security against an active adversary can be achieved by means of a linear distributed commitment and three corresponding auxiliary protocols: a commitment transfer protocol (CTP), a commitment sharing protocol (CSP) and a commitment multiplication protocol (CMP). A linear distributed commitment allows a player to commit to a secret, however, in contrast to its cryptographic counterpart, a distributed commitment is perfectly hiding and binding. A CTP allows to transfer a commitment for a secret from one player to another, a CSP allows to share a committed secret in a verifiable way such that the players will be committed to their shares, and a CMP allows to prove that three committed secrets $s, s^{\prime}$ and $s^{\prime \prime}$ satisfy the relation $s^{\prime \prime}=s s^{\prime}$, if this is indeed the case. These protocols allow to modify the passively secure MPC protocol, sketched in Section 3.3 in such a way that at every stage of the MPC every player is committed to its current intermediary results. This guarantees detection of dishonest behaviour and thus security against an active adversary. It is straightforward to verify that the field based solutions of [13] can be extended

\footnotetext{
${ }^{5}$ Perfect security requires a strongly-multiplicative span program, while an (ordinary) multiplicative span program is sufficient for unconditional security (see Section 3.4).

${ }^{6}$ Note that the corresponding number multiplication can efficiently be computed using standard "double and add", requiring only black-box access to the addition in $R$.
} 
to our more general setting of MPC over an arbitrary ring (please consult the full version [16] for a more detailed description). As in [13], the perfectly secure CMP requires a strongly multiplicative span program whereas an ordinary span program suffices for unconditional security.

\subsection{Threshold Black-Box MPC}

Consider a threshold adversary structure $\mathcal{A}_{t, n}=\{A \subseteq \mathcal{P}:|A| \leq t\}$ with $0<t<n$.

Proposition 1. Let $\Lambda$ be a commutative ring with 1 . Assume there exist units $\omega_{1}, \ldots, \omega_{n} \in \Lambda$ such that all pairwise differences $\omega_{i}-\omega_{j}(i \neq j)$ are invertible as well. Then there exists a span program $\mathcal{M}=(\Lambda, M, \psi, \varepsilon)$ for $\mathcal{A}_{t, n}$ of size $n$, which is (strongly) multiplicative if and only if $t<n / 2(t<n / 3)$ : the $i$-th row of $M$ is simply $\left(1, \omega_{i}, \omega_{i}^{2}, \ldots, \omega_{i}^{t}\right)$, labeled by $P_{i}$, and $\varepsilon=(1,0, \ldots, 0)^{T} \in \Lambda^{t+1}$.

The resulting secret sharing scheme (with the secret and shares sampled from a $\Lambda$-module $G$ ), formally coincides with the well known Shamir scheme [36], except that the interpolation points $\omega_{1}, \ldots, \omega_{n}$ have to be carefully chosen (from 1 ). The security of this generalized Shamir scheme has been proven in [18 17]. A full proof of Proposition 11 that includes the claim concerning the (strong) multiplication property can be found in the full version [16].

To achieve black-box MPC over an arbitrary finite ring $R$, it suffices, by Corollary 1 to have a (strongly multiplicative) ISP for $\mathcal{A}_{t, n}$. Unfortunately, the $\operatorname{ring} \Lambda=\mathbb{Z}$ does not fulfill the assumption of Proposition 1 (except for $n=1$ ), and hence Proposition 1 does not provide the desired ISP. However, by Lemma 1 below, it is in fact sufficient to provide a span program over an extension ring $\Lambda$ of $\mathbb{Z}$, as it guarantees that any such span program can be "projected" to an ISP.7 The remaining gap is then closed in Lemma 2 by exhibiting an extension $\operatorname{ring} \Lambda$ of $\mathbb{Z}$ that satisfies the assumption of Proposition 1

Lemma 1. Let $f(X) \in \mathbb{Z}[X]$ be a monic, irreducible polynomial of non-zero degree $m$, and let $\Lambda$ be the extension ring $\Lambda=\mathbb{Z}[X] /(f(X))$ of $\mathbb{Z}$. Then, any span program $\mathcal{M}$ over $\Lambda$ can be (efficiently) transformed into an integer span program $\overline{\mathcal{M}}$ for the same adversary structure such that $\operatorname{size}(\overline{\mathcal{M}})=m \cdot \operatorname{size}(\mathcal{M})$. Furthermore, if $\mathcal{M}$ is (strongly) multiplicative then this also holds for $\overline{\mathcal{M}}$.

The first part of this lemma appeared in [15]. A full proof of Lemma 1] also covering the multiplication property, can be found in the full version [16]. For a proof of Lemma 2 below we refer to [18].

\footnotetext{
7 Alternatively, one could also "lift" $R$ to an extension ring $S \supseteq R$ which can be seen as an algebra over $\Lambda \supseteq \mathbb{Z}$, and then do the MPC over $S$, using some mechanism that ensures that the inputs come from the smaller ring $R$. This approach, which has also been used in [18] in the context of secret sharing over arbitrary Abelian groups, would lead to a somewhat more efficient implementation of the MPC protocols; however, we feel that our approach serves better conceptual simplicity.
} 
Lemma 2. Consider the polynomials $\omega_{i}(X)=1+X+\cdots+X^{i-1} \in \mathbb{Z}[X]$ for $i=1, \ldots, n$. Then $\omega_{1}(X), \ldots, \omega_{n}(X)$ and all pairwise differences $\omega_{i}(X)-\omega_{j}(X)$ $(i \neq j)$ are invertible modulo the cyclotomic polynomial $\Phi_{q}(X)=1+X+\cdots+$ $X^{q-1} \in \mathbb{Z}[X]$, where $q$ is a prime greater than $n$.

Hence, if $t<n / 3$ then by Proposition 1 there exists a strongly-multiplicative span program $\mathcal{M}$ for $\mathcal{A}_{t, n}$ over the extension ring $\Lambda=\mathbb{Z}[X] /\left(\Phi_{q}(X)\right)$ where $q>n$. The size of $\mathcal{M}$ is $n$, and $q$ can be chosen linear in $n$ by Bertrand's Postulate. Together with Lemma 1 this implies a strongly-multiplicative ISP of size $O\left(n^{2}\right)$, and hence Corollary 1 yields

Corollary 2. For $t<n / 3$, there exists an $\mathcal{A}_{t, n}$-secure black-box MPC protocol to evaluate any arithmetic circuit $C$ over an arbitrary finite ring $R$.

A threshold ISP of size $O(n \log n)$ was presented in [15] (and proven optimal). As this construction too is related to Shamir's scheme, it is not hard to see that also this ISP is (strongly) multiplicative if and only if $t<n / 2(t<n / 3)$. Hence, it gives rise to another instantiation of the MPC protocol claimed in Corollary 2] Its communication complexity turns out to coincide asymptotically with the classical protocols of $[6,2 \mid 22]$, up to a possible loss of a factor $\log n$, which is due to the fact that over large fields there exist threshold span programs of size $n$. Furthermore, our protocol is compatible with improvements to the communication complexity of non-black-box MPC over fields [27,26].

\section{Constant-Round Protocols}

In this section we present constant-round MPC protocols over arbitrary rings. Our motivation is twofold. First, we complement the results of the previous section by showing that they carry over in their full generality to the constant-round setting. This does not immediately follow from previous work in the area. Second, we point out some improvements and simplifications to previous constant-round techniques, which also have relevance to the special case of fields. In particular, we obtain constant-round protocols for small-width branching programs and balanced formulas in which the communication complexity is nearly linear in their size.

\subsection{Randomizing Polynomials over Rings}

The results of the previous section may be viewed as providing a general "compiler", taking a description of an arithmetic circuit $C$ over some ring $R$ and producing a description of an MPC protocol for the functionality prescribed by $C$. While the communication complexity of the resultant protocol is proportional to the size of $C$, its round complexity is proportional to its multiplicative depth 8

\footnotetext{
${ }_{8}$ Multiplicative depth is defined similarly to ordinary circuit depth, except that addition gates and multiplications by constant do not count.
} 
In particular, constant-degree polynomials over $R$ can be securely evaluated in a constant number of rounds using black-box access to $R$. The notion of randomizing polynomials, introduced in [28], provides a convenient framework for representing complex functions as low-degree polynomials, thereby allowing their round-efficient secure computation. In the following we generalize this notion to apply to any function $f: R^{n} \rightarrow D$, where $R$ is an arbitrary ring and $D$ is an arbitrary set 9

A randomizing polynomials vector over the $\operatorname{ring} R$ is a vector $p=\left(p_{1}, \ldots, p_{s}\right)$ of $s$ multivariate polynomials over $R$, each acting on the same $n+m$ variables $x=\left(x_{1}, \ldots, x_{n}\right)$ and $r=\left(r_{1}, \ldots, r_{m}\right)$. The variables $x$ are called inputs and $r$ are called random inputs. The complexity of $p$ is the total number of inputs and outputs (i.e., $s+n+m$ ). Its degree is defined as the maximal degree of its $s$ entries, where both ordinary inputs and random inputs (but not constants) count towards the degree 10

Representation of a function $f$ by $p$ is defined as follows. For any $x \in R^{n}$, let $P(x)$ denote the output distribution of $p(x, r)$, induced by a uniform choice of $r \in R^{m}$. Note that for any input $x, P(x)$ is a distribution over $s$-tuples of ring elements. We say that $p$ represents a function $f$ if the output distribution $P(x)$ is "equivalent" to the function value $f(x)$. This condition is broken into two requirements, correctness and privacy, as formalized below.

Definition 4. A randomizing polynomials vector $p(x, r)$ is a said to represent the function $f: R^{n} \rightarrow D$ if the following requirements hold:

- CORRECTNESS. There exists an efficien 11 reconstruction algorithm which, given only a sample from $P(x)$, can correctly compute the output value $f(x)$.

- PRIVACY. There exists an efficient simulator which, given the output value $f(x)$, can emulate the output distribution $P(x)$.

$W e$ will also consider the relaxed notion of $\delta$-correct randomizing polynomials, where the reconstruction algorithm is allowed to output "don't know" with probability $\delta$ (but otherwise must be correct).

The application of randomizing polynomials to secure computation, discussed in [28], is quite straightforward. Given a representation of $f(x)$ by $p(x, r)$, the secure computation of $f$ can be reduced to the secure computation of the randomized function $P(x)$. The latter, in turn, reduces to the secure computation of the deterministic function $p^{\prime}\left(x, r^{1}, \ldots, r^{a}\right) \stackrel{\text { def }}{=} p\left(x, r^{1}+\ldots+r^{a}\right)$, where $a$ is the size of some set $A \notin \mathcal{A}$, by assigning each input vector $r^{j}$ to a distinct player in $A$ and instructing it to pick $r^{j}$ at random. Note that the degree of $p^{\prime}$ is the same as that of $p$. Moreover, if the reconstruction procedure associated with $p$ requires only black-box access to $R$, then this property is maintained by the reduction.

${ }^{9}$ In this section the parameter $n$ is used to denote an input length parameter rather than the number of players. The input, taken from $R^{n}$, may be arbitrarily partitioned among any number of players.

${ }^{10}$ It is crucial for the MPC application that random inputs count towards the degree.

11 The efficiency requirement can only be meaningfully applied to a family of randomizing polynomials, parameterized by the input size $n$ and the ring $R$. 
Hence, using the results of the previous section, the problem of getting roundefficient MPC over rings reduces to that of obtaining low-degree representations for the functions of interest.

In the following we describe two constructions of degree- 3 randomizing polynomials over rings, drawing on techniques from [29]3].

\subsection{Branching Programs over Rings}

Branching programs are a useful and well-studied computational model. In particular, they are stronger than the formula model (see Section 4.3). We start by defining a general notion of branching programs over an arbitrary ring $R$.

Definition 5. A branching program BP on inputs $x=\left(x_{1}, \ldots, x_{n}\right)$ over $R$ is defined by: (1) a DAG (directed acyclic graph) $G=(V, E)$; (2) a weight function $w$, assigning to each edge a degree-1 polynomial over $R$ in the input variables. It is convenient to assume that $V=\{0,1, \ldots, \ell\}$, where $\ell$ is referred to as the size of $B P$, and that for each edge $(i, j) \in E$ it holds that $i<j$. The function computed by $B P$ is defined as follows. For each directed path $\phi=\left(i_{1}, i_{2}, \ldots, i_{k}\right)$ in $G$, the weight of $\phi$ is defined to be the product $w\left(i_{1}, i_{2}\right) \cdot w\left(i_{2}, i_{3}\right) \cdot \ldots \cdot w\left(i_{k-1}, i_{k}\right)$ (in the prescribed order). For $i<j$, we denote by $W(i, j)$ the total weight of all directed paths from $i$ to $j$ (viewed as a function of $x$ ). Finally, the function $f: R^{n} \rightarrow R$ computed by $B P$ is defined by $f(x)=W(0, \ell)(x)$. We refer to $W(0, \ell)$ as the output of $B P$.

Note that, using a simple dynamic programming algorithm, the output of $B P$ can be evaluated from its edge weights using $O(|E|)$ black-box ring operations.

To represent a branching program by randomizing polynomials, we rely on a recent construction from [29. However, applying this construction to general rings requires a different analysis. In particular, the original analysis relies on properties of the determinant which do not hold in general over non-commutative rings. Below we provide a more combinatorial interpretation of this construction, which may be of independent interest.

How to GARBLE A BRANCHING PROGRAM. Given a branching program $B P=$ $(G, w)$ of size $\ell$, we define a randomized procedure producing a "garbled" branching program $\tilde{B P}=(\tilde{G}, \tilde{w})$ of the same size $\ell$. The graph $\tilde{G}$ will always be the complete DAG, i.e., each $(i, j)$ where $0 \leq i<j \leq \ell$ is an edge in $\tilde{G}$. We will sometimes also view $G$ as a complete graph, where $w(i, j)=0$ if $(i, j)$ is not originally an edge. The randomization of $B P$ proceeds in two phases.

Main phase. Let $r_{i j}, 0 \leq i<j<\ell$, be $\left(\begin{array}{l}\ell \\ 2\end{array}\right)$ random and independent ring elements. Each $r_{i j}$ naturally corresponds to an edge. The main randomization phase modifies each original weight $w(i, j)$ as follows: first, if $j<\ell$, it increases it by $r_{i j}$. Then, regardless of whether $j=\ell$, it decreases it by $r_{i h} \cdot w(h, j)$ for each $h$ lying strictly between $i$ and $j$. That is, the updated weights $w^{\prime}(i, j)$ obtained at the end of this phase are defined by

$$
w^{\prime}(i, j)= \begin{cases}w(i, j)+r_{i j}-\sum_{h=i+1}^{j-1} r_{i h} \cdot w(h, j), & j<\ell \\ w(i, j)-\sum_{h=i+1}^{j-1} r_{i h} \cdot w(h, j), & j=\ell\end{cases}
$$


Note that each $w^{\prime}(i, j)$ is a degree-2 polynomial in the inputs $x$ and the random inputs $r_{i j}$.

Cleanup phase. In the main phase the weights of the edges entering $\ell$ were not fully randomized. In some sense, these edges served as "garbage collectors" for the randomization of the remaining edges. To eliminate the unwanted residual information about $x$, the following operation is performed. Let $r_{1}^{\prime}, \ldots, r_{\ell-1}^{\prime}$ be independent random ring elements. The new weights $\tilde{w}$ are the same as $w^{\prime}$ for $(i, j)$ such that $j<\ell$, and else are defined by:

$$
\tilde{w}(i, \ell)= \begin{cases}w^{\prime}(i, \ell)-\sum_{j=i+1}^{\ell-1} w^{\prime}(i, j) \cdot r_{j}^{\prime}, & i=0 \\ w^{\prime}(i, \ell)+r_{i}^{\prime}-\sum_{j=i+1}^{\ell-1} w^{\prime}(i, j) \cdot r_{j}^{\prime}, & i>0\end{cases}
$$

Note that the weights $\tilde{w}(i, \ell)$ are degree-3 polynomials in $x, r, r^{\prime}$ and the remaining weights are all of degree 2 . Still, each weight $\tilde{w}$ is of degree 1 in $x$, and hence any fixed choice of $r, r^{\prime}$ indeed makes $\tilde{B P}$ a branching program according to our definition.

We define a randomizing polynomials vector $p\left(x, r, r^{\prime}\right)$ representing $B P$ by the concatenation of all $\left(\begin{array}{c}\ell+1 \\ 2\end{array}\right)$ weights $\tilde{w}$. It has degree 3 and complexity $O\left(\ell^{2}\right)$. It can be evaluated using $O(|E| \ell)$ ring operations assuming that each original weight $w$ depends on a single input variable. We prove its correctness and privacy.

Correctness. It suffices to show that on any input $x$, the value of $\tilde{B P}$ equals that of $B P$, for any choice of $r, r^{\prime}$. For this, it suffices to show that the positive and negative contributions of each random input cancel each other. Consider the effect of a specific random input $r_{i j}$ in the main phase. It is involved in two types of operations: (1) it is added to $w(i, j)$; and $(2) r_{i j} \cdot w(j, k)$ is subtracted from each weight $w(i, k)$ such that $k>j$. We now compare the contribution of (1) and $(2)$ to the output $W(0, \ell)$. Since $(1)$ affects exactly those paths that traverse the edge $(i, j)$, the positive contribution of $(1)$ is

$$
W(0, i) \cdot r_{i j} \cdot W(j, \ell) .
$$

(Note that, by the distributive law, the above expression covers exactly all directed paths from 0 to $\ell$ passing through $(i, j)$.) Similarly, the negative contribution of $(2)$ is:

$$
\begin{aligned}
\sum_{k>j} W(0, i) \cdot\left(r_{i j} \cdot w(j, k)\right) \cdot W(k, \ell) & =W(0, i) \cdot r_{i j} \sum_{k>j} w(j, k) \cdot W(k, \ell) \\
& =W(0, i) \cdot r_{i j} \cdot W(j, \ell)
\end{aligned}
$$

Hence, the positive and negative contributions of each $r_{i j}$ exactly cancel each other, as required. A similar argument applies to the cleanup phase operations involving $r_{i}^{\prime}$ (details omitted). To conclude, it suffices for the reconstruction procedure to evaluate the garbled branching program $\tilde{B P}\left(x, r, r^{\prime}\right)$, which requires $O\left(\ell^{2}\right)$ ring operations.

Privacy. We argue that, for any fixed $x$, the distribution of $\tilde{w}$ induced by the random choice of $r, r^{\prime}$ is uniform among all weight assignments having the same 
output value as $B P$ on $x$. First, note that the number of possible choices of $r, r^{\prime}$ is $|R|^{\left(\begin{array}{l}\ell \\ 2\end{array}\right)+(\ell-1)}$, which is exactly equal to $|R|^{\left(\begin{array}{c}\ell+1 \\ 2\end{array}\right)-1}$, the number of possible weight assignments in each output class 12 It thus suffices to prove that, for any fixed weight assignment $w$, the effect of $r, r^{\prime}$ on $w$ (as a function from $R^{\left(\begin{array}{c}\ell+1 \\ 2\end{array}\right)-1}$ to $\left.R^{\left(\begin{array}{c}\ell+1 \\ 2\end{array}\right)}\right)$ is one-to-one. Consider two distinct vectors of random inputs, $\left(r, r^{\prime}\right)$ and $\left(\hat{r}, \hat{r}^{\prime}\right)$. Order each of them by first placing the $r_{i j}$ entries in increasing lexicographic order and then the $r_{i}^{\prime}$ entries in decreasing order. Consider the first position where the two ordered lists differ. It is not hard to verify that if the first difference is $r_{i j} \neq \hat{r}_{i j}$, where $j<\ell$, then the weight of $(i, j)$ will differ after the main phase. (Note that since $j<\ell$, this weight is untouched in the cleanup phase.) The second case, where the first difference is $r_{i}^{\prime} \neq \hat{r}_{i}^{\prime}$, is similar. In this case the two random inputs will induce the same change to the weight of $(i, \ell)$ in the main phase, and a different change in the cleanup phase. Thus, the garbled weight function is indeed uniformly random over its output class. Given the above, a simulator may proceed as follows. On output value $d \in R$, the simulator constructs a branching program $B P$ with $w(0, \ell)=d$ and $w(i, j)=0$ elsewhere, and outputs a garbled version $\tilde{B P}$ of $B P$.

Combining the above with the results of the previous section, we have:

Theorem 2. Let BP be a branching program over a black-box ring $R$, where BP has size $\ell$ and $m$ edges. Then BP admits a perfectly secure MPC protocol, communicating $O\left(\ell^{2}\right)$ ring elements and performing $O(m \ell)$ ring operations (ignoring polynomial dependence on the number of players). The protocol may achieve an optimal security threshold, and its exact number of rounds corresponds to that of degree-3 polynomials.

TRADING COMMUNICATION FOR ROUNDS. For large branching programs, the quadratic complexity overhead of the previous construction may be too costly. While this overhead somehow seems justified in the general case, where the description size of $B P$ may also be quadratic in its size $\ell$, one can certainly expect improvement in the typical case where $B P$ has a sparse graph. A useful class of such branching programs are those that have a small width. $B P$ is said to have length $a$ and width $b$ if the vertices of its graph $G$ can be divided into $a$ levels of size $\leq b$ each, such that each edge connects two consecutive levels. For instance, for any binary regular language, the words of length $n$ can be recognized by a constant-width length- $n$ branching program over $\mathbb{Z}_{2}$ (specifically, the width is equal to the number of states in the corresponding automaton).

For the case of small-width branching programs, we can almost eliminate the quadratic overhead at the expense of a moderate increase in the round complexity. We use the following recursive decomposition approach. Suppose that the length of $B P$ is broken into $s$ segments of length $a / s$ each. Moreover, suppose that in each segment all $b^{2}$ values $W(i, j)$ such that $i$ is in the first level of that

12 Indeed, among the $|R|^{\left(\begin{array}{c}\ell+1 \\ 2\end{array}\right)}$ possible ways of fixing the weights, there is an equal representation for each output. A bijection between the weight functions of two output values $d_{1}, d_{2}$ can be obtained by adding $d_{1}-d_{2}$ to the weight of $(0, \ell)$. 
segment and $j$ is in the last level are evaluated. Then, the output of $B P$ can be computed by a branching program $B P^{\prime}$ of length $s$ and width $b$, such that the edge weights of $B P^{\prime}$ are the $b^{2} s$ weights $W(i, j)$ as above. Thus, the secure computation of $B P$ can be broken into two stages: first evaluate in parallel $b^{2} s$ branching programs of length $a / s$ and width $b$ each, producing the weights $W(i, j)$, and then use these weights as inputs to the length- $s$, width- $b$ branching program $B P^{\prime}$, producing the final output. This process requires to hide the intermediate results produced by the first stage, which can be done with a very small additional overhead. In fact, a careful implementation (following [3]) allows the second stage to be carried out using only a single additional round of broadcast.

If the width $b$ of $B P$ is constant, an optimal choice for $s$ is $s=O\left(a^{2 / 3}\right)$, in which case the communication complexity of each of the two stages becomes $O\left(a^{4 / 3}\right)$. This is already a significant improvement over the $O\left(a^{2}\right)$ complexity given by Theorem 2 Moreover, by recursively repeating this decomposition and tuning the parameters, the complexity can be made arbitrarily close to linear while maintaining a (larger) constant number of rounds. In particular, this technique can be used to obtain nearly-linear perfect constant-round protocols for iterated ring multiplication or for Yao's millionaires' problem [37], both of which admit constant-width linear-length branching programs.

\subsection{Arithmetic Formulas}

An arithmetic formula over a ring $R$ is defined by a rooted binary tree, whose leaves are labeled by input variables and constants (more generally, by degree-1 polynomials), and whose internal nodes, called gates, are labeled by either ' +' (addition) or ' $x$ ' (multiplication). If $R$ is non-commutative, the children of each multiplication node must be ordered. A formula is evaluated in a gate-by-gate fashion, from the leaves to the root. Its size is defined as the number of leaves and its depth as the length of the longest path from the root to a leave. A formula is balanced if it forms a complete binary tree.

We note that the branching program model is strictly stronger than the formula model. In particular, any formula (even with gates of unbounded fanin) can be simulated by a branching program of the same size. Thus, the results from Section 4.2 apply to formulas as well.

We combine a complexity result due to Cleve [12] with a variant of a randomization technique due to Beaver 3] (following Kilian [31] and Feige et al. [20]) to obtain an efficient representation of formulas by degree- 3 randomizing polynomials. If the formula is balanced, the complexity of this representation can be made nearly linear in the formula size. However, in contrast to the previous construction, the current one will not apply to a black-box ring $R$ and will not offer perfect correctness. Still, for the case of balanced arithmetic formulas, it can provide better efficiency.

$\overline{13}$ It is possible to avoid the $b^{2}$ overhead by modifying the garbled branching program construction so that all weights $W(i, j)$ in each segment are evaluated at once. 
From FORMUlas TO ITERATED MATRIX PRODUCT. In [12, it is shown that an arithmetic formula of depth $d$ over an arbitrary ring $R$ with 1 can be reduced to the iterated product of $O\left(\left(2^{d}\right)^{1+\frac{2}{b}}\right)$ matrices of size $c=O\left(2^{b}\right)$ over $R$, for any constant $b$. Each of these matrices is invertible, and its entries contain only variables or constants 14 The output of the formula is equal to the top-right entry of the matrix product. Note that if the formula is balanced, then the total size of all matrices can be made nearly linear in the formula size.

Next, we consider the problem of randomizing an entry of an iterated matrix product as above. Having already established the possibility of constant-round MPC over black-box rings, we focus on efficiency issues and restrict our attention to the special case of fields. Indeed, the following construction does not apply to black-box rings (though may still apply with varied efficiency to non-field rings). In what follows we let $K$ denote a finite field, $K^{c \times c}$ the set of $c \times c$ matrices over $K$, and $\mathrm{GL}_{c}(K)$ the group of invertible $c \times c$ matrices over $K$.

RANDOMIZING AN ITERATED PRODUCT OF INVERTIBLE MATRICES. To represent an iterated matrix product by degree-3 randomizing polynomials, we modify a randomization technique from [3. See [16] for a proof of the next proposition.

Proposition 2. Let $M_{2}, \ldots, M_{k-1} \in \mathrm{GL}_{c}(K)$, let $\hat{M}_{1}$ be a nonzero row vector and $\hat{M}_{k}$ a nonzero column vector. Suppose that at most a $(\delta / 2 k)$-fraction of the $c \times c$ matrices over $K$ are singular. Then,

$$
\left(\hat{M}_{1} S_{1}, S_{2} S_{1}, S_{2} M_{2} S_{3}, S_{4} S_{3}, S_{4} M_{3} S_{5}, \ldots, S_{2 k-2} S_{2 k-3}, S_{2 k-2} \hat{M}_{k}\right),
$$

where $S_{1}, \ldots, S_{2 k-2}$ are uniformly random matrices, is a $\delta$-correct degree-3 representation for the iterated product $\hat{M}_{1} M_{2} \cdots M_{k-1} \hat{M}_{k}$.

Note that if the field $K$ is small, the correctness probability can be boosted by working over an extension field (thereby increasing the probability of picking invertible matrices).

By choosing $\hat{M}_{1}$ and $\hat{M}_{k}$ to be unit vectors, Proposition 2 can be used to represent a single entry in an iterated product of invertible matrices, as required for applying Cleve's reduction. Thus, we have:

Theorem 3. Let $F$ be an arithmetic formula of depth $d$ over a finite field $K$. Then, $F$ admits a constant-round MPC protocol communicating $2^{d+O(\sqrt{d})}$ field elements (i.e., $s \cdot 2^{O(\sqrt{\log s})}$ elements if $F$ is a balanced formula of size $\left.s\right)$. The protocol can either have a minimal round complexity (corresponding to degree-3 polynomials) with $O\left(|K|^{-1}\right)$ failure probability, or alternatively achieve perfect correctness and privacy in an expected constant number of rounds (where the expected overhead to the number of rounds can be made arbitrarily small).

\section{Application: Securely Computing the MAX Function}

Aside from its theoretical value, the study of MPC over non-field rings is motivated by the possibility of embedding useful computation tasks into their richer

$\overline{14}$ The requirement that $R$ has 1 can be dispensed with by using an appropriate extension of $R$. 
structure. In this section we demonstrate the potential usefulness of this approach by describing an application to the round-efficient secure computation of the maximum function.

Suppose there are $n$ players, where each player $P_{i}$ holds an integer $y_{i}$ from the set $\{0,1, \ldots, M\}$. (We consider $M$ to be a feasible quantity.) Our goal is to design a protocol for securely evaluating $\max \left(y_{1}, \ldots, y_{n}\right)$ with the following optimization criteria in mind. First, we would like the round complexity to be as small as possible. Second, we want to minimize the communication complexity subject to the latter requirement.

Our solution proceeds as follows. Let $k$ be a (statistical) security parameter, and fix a ring $R=\mathbb{Z}_{Q^{M}}$ where $Q$ is a $k$-bit prime. We denote the elements of $R$ by $1,2, \ldots, Q^{M}=0$. Consider the degree-2 randomizing polynomial

$$
p\left(x_{1}, \ldots, x_{n}, r_{1}, \ldots, r_{n}\right)=\sum_{i=1}^{n} r_{i} x_{i}
$$

over $R$. It is not hard to verify that: (1) the additive group of $R$ has exactly $M+1$ subgroups, and these subgroups are totally ordered with respect to containment 15 and (2) the output distribution $P\left(x_{1}, \ldots, x_{n}\right)$ is uniform over the maximal (i.e., largest) subgroup generated by an input $x_{i}$. Specifically, $P\left(x_{1}, \ldots, x_{n}\right)$ is uniform over the subgroup generated by $Q^{j}$, where $j$ is the maximal integer from $\{0,1, \ldots, M\}$ such that $Q^{j}$ divides all $x_{i}$.

We are now ready to describe the protocol. First, each player $i$ maps its input $y_{i}$ to the ring element $x_{i}=Q^{M-y_{i}}$. Next, the players securely sample an element $z$ from the output distribution $P\left(x_{1}, \ldots, x_{n}\right)$. This task can be reduced to the secure evaluation of a deterministic degree-2 polynomial over $R$ (see Section 4.1). Finally, the output of the computation is taken to be the index of the minimal subgroup of $R$ containing $z$; i.e., the output is 0 if $z=0$ and otherwise it is $M-\max \left\{j: Q^{j}\right.$ divides $\left.z\right\}$. Note that the value of $z$ reveals no information about the inputs $y_{i}$ except what follows from their maximum, and the protocol produces the correct output except with probability $1 / Q \leq 2^{-(k-1)}$. We stress that an active adversary (or malicious players) cannot gain any advantage by picking "invalid" inputs $x_{i}^{*}$ to the evaluation of $P$. Indeed, any choice of $x_{i}^{*}$ is equivalent to a valid choice of $x_{i}$ generating the same subgroup.

We turn to analyze the protocol's efficiency. Recall that our main optimization criterion was the round complexity. The protocol requires the secure evaluation of a single degree-2 polynomial over $R$. Using off-the-shelf MPC protocols (adapted to the ring $R$ as in Section 3 ), this requires fewer rounds than evaluating degree- 3 polynomials or more complex functions 16 The communication complexity of the protocol is linear in $M$ and polynomial in the number of players. In [16] we discuss several alternative approaches for securely evaluating the

$\overline{15}$ This should be contrasted with the field of the same cardinality, which has $2^{M}$ partially ordered additive subgroups.

16 The exact number of rounds being saved depends on the specific setting, e.g. on whether a broadcast channel is available. 
maximum function. All of these alternatives either require more rounds, require a higher communication complexity (quadratic in $M$ ), or fail to remain secure against an active adversary.

Acknowledgements. We would like to thank Ivan Damgård and Tal Rabin for helpful discussions.

\section{References}

1. J. Bar-Ilan and D. Beaver. Non-cryptographic fault-tolerant computing in a constant number of rounds of interaction. In Proc. of 8th PODC, pp. 201-209, 1989.

2. D. Beaver. Efficient multiparty protocols using circuit randomization. In Proc. of CRYPTO '91, LNCS 576, pp. 420-432, 1991.

3. D. Beaver. Minimal-latency secure function evaluation. In Proc. of EUROCRYPT '00, LNCS 1807, pp. 335-350, 2000.

4. D. Beaver, J. Feigenbaum, J. Kilian, and P. Rogaway. Security with low communication overhead (extended abstract). In Proc. of CRYPTO '90, LNCS 537, pp. 62-76, 1990.

5. D. Beaver, S. Micali, and P. Rogaway. The round complexity of secure protocols (extended abstract). In Proc. of 22nd STOC, pp. 503-513, 1990.

6. M. Ben-Or, S. Goldwasser, and A. Wigderson. Completeness theorems for noncryptographic fault-tolerant distributed computation. In Proc. of 20th STOC, pp. 1-10, 1988.

7. R. Canetti. Security and composition of multiparty cryptographic protocols. In $J$. of Cryptology, 13(1):143-202, 2000.

8. R. Canetti. Universally Composable Security: A New Paradigm for Cryptographic Protocols. In Proc. of 42nd FOCS, pp. 136-145, 2001.

9. R. Canetti, U. Feige, O. Goldreich and M. Naor. Adaptively secure computation. In Proc. of 28th STOC, pp. 639-648, 1996.

10. D. Chaum, C. Crepeau, and I. Damgård. Multiparty unconditional secure protocols. In Proc. of 20th STOC, pp. 11-19, 1988.

11. C. Cachin, J. Camenisch, J. Kilian, and J. Muller. One-round secure computation and secure autonomous mobile agents. In Proc. of 27th ICALP, pp. 512-523, 2000.

12. R. Cleve. Towards Optimal Simulations of Formulas by Bounded-Width Programs. In Computational Complexity 1: 91-105, 1991.

13. R. Cramer, I. Damgård, and U. Maurer. General secure multi-party computation from any linear secret-sharing scheme. In Proc. of EUROCRYPT '00, LNCS 1807, pp. 316-334, 2000.

14. R. Cramer, I. Damgård, and J. Nielsen. Multiparty computation from threshold homomorphic encryption. In Proc. of EUROCRYPT '01, LNCS 2045, pp. 280-299, 2001.

15. R. Cramer and S. Fehr. Optimal black-box secret sharing over arbitrary Abelian groups. In Proc. of CRYPTO '02, LNCS 2442, 272-287, 2002.

16. R. Cramer, S. Fehr, Y. Ishai, and E. Kushilevitz. Efficient multi-party computation over rings. Cryptology ePrint Archive, Report 2003/030, 2003.

17. A. De Santis, Y. Desmedt, Y. Frankel, and M. Yung. How to share a function securely. In Proc. of 26th STOC, pp. 522-533, 1994. 
18. Y. G. Desmedt and Y. Frankel. Homomorphic zero-knowledge threshold schemes over any finite Abelian group. In SIAM Journal on Discrete Mathematics, 7(4):667679, 1994.

19. M. Fitzi, M. Hirt, and U. Maurer. Trading correctness for privacy in unconditional multi-party computation. In Proc. of CRYPTO '98, LNCS 1462, pp. 121-136, 1998.

20. U. Feige, J. Kilian, and M. Naor. A minimal model for secure computation. In Proc. of 26th STOC, pp. 554-563, 1994.

21. M. Fitzi and U. Maurer Efficient Byzantine agreement secure against general adversaries. In Proc. of DISC '98, LNCS 1499, pp. 134-148, 1998.

22. M. Franklin and M. Yung. Communication complexity of secure computation. In Proc. of 24th STOC, pp. 699-710, 1992.

23. R. Gennaro, M. O. Rabin, and T. Rabin. Simplified VSS and fast-track multiparty computations with applications to threshold cryptography. In Proc. of 17th PODC, pp. 101-111, 1998.

24. O. Goldreich, S. Micali, and A. Wigderson. How to play any mental game (extended abstract). In Proc. of 19th STOC, pp. 218-229, 1987.

25. M. Hirt and U. Maurer. Complete characterization of adversaries tolerable in secure multi-party computation (extended abstract). In Proc. of 16th PODC, 1997, pp. $25-34$.

26. M. Hirt and U. Maurer. Robustness for free in unconditional multi-party computation. In Proc. of CRYPTO '01, LNCS 2139, pp. 101-118, 2001.

27. M. Hirt, U. Maurer, and B. Przydatek. Efficient secure multi-party computation. In Proc. of ASIACRYPT '00, LNCS 1976, pp. 143-161, 2000.

28. Y. Ishai and E. Kushilevitz. Randomizing polynomials: A new representation with applications to round-efficient secure computation. In Proc. of 41 th FOCS, pp. 294-304, 2000.

29. Y. Ishai and E. Kushilevitz. Perfect constant-round secure computation via perfect randomizing polynomials. In Proc. of 29th ICALP, pp. 244-256, 2002.

30. M. Karchmer and A. Wigderson. On span programs. In Proc. of 8th Conference on Structure in Complexity Theory, pp. 102-111, 1993.

31. J. Kilian. Founding cryptography on oblivious transfer. In Proc. of 20th STOC, pp. 20-31, 1988.

32. Y. Lindell. Parallel coin-tossing and constant-round secure two-party Computation. In Proc. of CRYPTO '01, LNCS 2139, pp. 171-189, 2001.

33. M. Naor, and K. Nissim. Communication Preserving Protocols for Secure Function Evaluation. In Proc. of 33rd STOC, pp. 590-599, 2001.

34. R. Ostrovsky and M. Yung. How to withstand mobile virus attacks. In Proc. of 10th PODC, pp. 51-59, 1991.

35. T. Sandler, A. Young, and M. Yung. Non-interactive cryptocomputing for $\mathrm{NC}^{1}$. In Proc. of 40th FOCS, pp. 554-567, 1999.

36. A. Shamir. How to share a secret. CACM, 22(11):612-613, 1979.

37. A. C. Yao. Protocols for secure computations. In Proc. of 23th FOCS, pp. 160-164, 1982.

38. A. C. Yao. How to generate and exchange secrets. In Proc. of 27th FOCS, pp. 162-167, 1986. 